\title{
Fragilité des projets d'élevage familiaux dans les exploitations de polyculture-élevage au Burkina Faso
}

\author{
Barkwendé Jéthro Delma ${ }^{1, *}$, Valérie Bougouma-Yameogo ${ }^{2}$, Hassan Bismarck Nacro ${ }^{2}$ et Eric Vall ${ }^{3}$ \\ ${ }^{1}$ Centre international de recherche-développement sur l'élevage en zone subhumide (CIRDES), 01 BP 454, Bobo-Dioulasso, Burkina Faso \\ ${ }^{2}$ Institut du développement rural (IDR), université polytechnique de Bobo-Dioulasso (UPB), 01 BP 1091, Bobo-Dioulasso, Burkina Faso \\ ${ }^{3}$ Centre international de recherche agronomique pour le développement (Cirad), UMR Selmet, 34398 Montpellier, France
}

\begin{abstract}
Résumé - Dans les exploitations de polyculture-élevage du Burkina Faso, les producteurs ont des difficultés à mettre en œuvre des projets d'élevage familiaux (PEF) qui répondent à leurs attentes en termes de revenus et de services. La présente étude vise à rechercher et à expliquer les causes de ces faiblesses. L'étude a été réalisée dans l'Ouest du Burkina Faso sur des PEF concernant des bovins. Elle a permis de montrer que tous les producteurs avaient des idées de PEF en tête. Un suivi a été réalisé sur des PEF de bovins de trait, de bovins à l'engraissement et de vaches laitières durant les étapes de conception puis d'exécution des PEF. Il en ressort que la majorité des PEF ont échoué avant même leur mise en œuvre, par manque de réalisme et de préparation. Ceux mis en place présentaient d'importantes faiblesses dans la maîtrise de l'alimentation, ce qui diminuait leur rentabilité. Ce diagnostic a permis d'établir les principes d'une démarche de conception pas à pas des PEF impliquant fortement le producteur, lui permettant de mieux calibrer et de mieux préparer le PEF, et de mieux gérer l'alimentation des animaux durant la mise en œuvre du PEF.
\end{abstract}

Mots clés : projets d'élevage familiaux / conception / alimentation / Burkina Faso

\begin{abstract}
Fragility of family livestock projects in mixed farming systems in Burkina Faso. In mixed farming systems in Burkina Faso, farmers have difficulty to implement family livestock projects (FLPs) meeting their expectations in terms of income and services. This study aims to investigate and to explain the causes of these weaknesses. The study was implemented in western Burkina Faso on cattle FLPs. It showed that all farmers had FLP ideas in mind. A monitoring was performed on draft animals, cattle for fattening and dairy cows FLPs during the design and implementation steps. It showed that the majority of FLPs failed even before their implementation, due to lack of preparation or unrealistic objectives. The implemented FLPs have important weaknesses concerning feeding control, which reduce their profitability. This diagnosis enabled us to set up the principles of a PEF step by step co-design method, to assist farmers to better prepare their FLP, to better manage animals feeding during their implementation and to better evaluate the outputs of the FLP.
\end{abstract}

Keywords: family livestock project / co-design / feeding / Burkina Faso

\section{Introduction}

Le secteur de l'élevage est en plein essor en Afrique de l'Ouest en raison de la demande croissante en produits animaux (Club du Sahel, 2008 ; McDermott et al., 2010 ; FAO et al., 2013 ; Kagone, 2001) et des multiples rôles qu'il joue dans le domaine social, économique et environnemental (Alary et al., 2011 ; Lebacq et al., 2013). Pourtant, dans les exploitations familiales de polyculture-élevage de la zone des savanes subhumides, la contribution de l'élevage à l'approvisionnement du marché en viande et en lait, à la génération de revenu

*Auteur de correspondance : delmabjethro@yahoo.fr. dans les exploitations ainsi qu'à la production de services (traction animale, fumure organique, épargne) n'est pas suffisante (De Ridder et al., 2015 ; Douxchamps et al., 2015). C'est notamment le cas au Burkina Faso, bien que l'élevage contribue à une part significative du produit intérieur brut $(18 \%$ ), qu'il soit pratiqué par plus de $80 \%$ de la population agricole, et qu'il représente une des principales activités de diversification et de sécurisation des revenus des exploitations agricoles familiales (MRA, 2012).

Deux principaux types de systèmes d'élevage bovins coexistent au Burkina Faso : le système extensif et mobile caractérisé par une faible utilisation d'intrants, et les systèmes en voie d'intensification dans lesquels les producteurs investissent plus de travail et de moyens financiers (achats 
d'aliments, stockage de fourrage, soins de santé, construction de bâtiments sommaires) pour accroître la productivité (Kagone, 2001). Dans cette dernière catégorie, on trouve trois principaux types de projets d'élevage familiaux (PEF) : ceux concernant les bovins de trait (BdT), ceux concernant les vaches laitières (VL), et ceux concernant les bovins à l'engraissement (BàE). Aujourd'hui, la demande des producteurs en appui à la conception, à la préparation et à la mise en œuvre de PEF n'est généralement pas couverte par les services de l'élevage. Ces derniers se limitent le plus souvent à une intervention dans le domaine vétérinaire. Or dans les démarches de conseil à l'élevage, qui à ce jour demeurent des opérations pilotes, les résultats ont montré qu'un appui dans la phase de conception et d'exécution permettait de mieux réussir un PEF (Awono Bessa et al., 2003 ; Vall et al., 2007) surtout dans des exploitations où la maîtrise technique et la capacité d'investissement étaient faibles et menaçaient la réussite des PEF (Kiéma et al., 2008).

Quelles sont les difficultés auxquelles se heurtent les producteurs pour atteindre les résultats qu'ils escomptent en matière de revenus (PEF, BàE et VL) ou de services (PEF, $\mathrm{BdT})$ ? Notre hypothèse est que les producteurs rencontrent des difficultés au cours de l'étape de conception de leur PEF, puis dans la maîtrise de l'alimentation au cours de sa mise en œuvre. Cette étude vise donc à analyser les causes d'échecs des PEF durant ces deux étapes.

\section{Matériel et méthodes}

L'étude a été conduite dans la province du Tuy, située en zone cotonnière à l'Ouest du Burkina Faso. Elle est caractérisée par une prédominance d'exploitations familiales de polyculture-élevage (coton, maïs, bovins), est soumise à un climat de type soudanien $(1000 \mathrm{~mm}$ de pluie/an) et présente une forte densité de population (40-50 hab/ $\mathrm{km}^{2}$ ) (Vall et al., 2006). Dans cette zone, de nombreuses exploitations ont recours à la traction animale et l'on trouve de plus en plus d'ateliers de VL et de BàE chez les producteurs, avec des possibilités d'écoulement des produits vers les villes de Houndé et Bobo-Dioulasso, voire au-delà, ce qui a justifié le choix de cette zone.

Pour mieux cerner les objectifs généraux des producteurs en matière d'élevage et pour repérer des PEF en préparation, une enquête préliminaire a été réalisée sur un échantillon de 40 exploitations de quatre villages du Tuy, réparties dans les trois principales catégories d'exploitations reconnues localement (Vall et al., 2006) : 15 agriculteurs, 13 agro-éleveurs et 12 éleveurs. Cette enquête a permis de préciser comment les producteurs entrevoyaient l'évolution de leur cheptel, de repérer les PEF bovins en préparation et de préciser les améliorations qu'ils envisageaient en matière d'infrastructures d'élevage et de pratiques de conduites alimentaires et sanitaires. L'enquête a permis d'identifier une douzaine de producteurs qui avaient des PEF en préparation (BdT, BàE ou VL) et étaient volontaires pour participer à un suivi de leur projet.

Sur la base de ces deux critères (PEF en préparation, volontariat), 12 porteurs de $\mathrm{PEF}$ (cinq BdT, quatre BàE et trois VL) ont participé à un suivi de leur PEF. Le travail a commencé par une enquête visant à caractériser le PEF prévisionnel initial (contexte, objectifs visés, modalités d'exécution du PEF : nombre d'animaux, alimentation et soins, charges et recettes escomptées...). Les PEF initiaux ont été étudiés pour repérer leurs forces et leurs faiblesses. Les porteurs des PEF ont ensuite été rencontrés à plusieurs reprises pour discuter d'ajustements possibles permettant de réduire leurs faiblesses. Ces allersretours ont abouti à des PEF prévisionnels, dont les points essentiels seront présentés dans les résultats.

Le travail a été prolongé par un suivi de l'exécution des PEF pendant six mois : engraissement et vente de bovins de janvier à mai 2014 pour les BàE, préparation à la campagne agricole de janvier à juin 2014 pour les BdT, production de lait en saison sèche de janvier à juin 2014 pour les VL. Durant la phase d'exécution, les PEF étaient visités tous les 15 jours jusqu'à leur terme (PEF réalisé). Au cours de chaque visite, l'équipe enregistrait les évolutions du PEF, discutait du PEF avec le producteur (réussite, difficultés...), collectait des données sur les animaux (poids vif, date de mise bas, intensité du travail pour les animaux de trait), les pratiques d'alimentation (durée de pâturage, pesées des offerts et des refus en fourrages et en aliments) et la trésorerie (recettes, dépenses). Au terme de l'exécution, une dernière visite a permis d'établir un bilan et d'aborder les perspectives avec le producteur.

Les mesures bimensuelles, réalisées sur l'état des animaux et sur les pratiques d'alimentation (offerts et ingérés), ont permis de suivre l'évolution de la couverture des besoins des animaux en énergie (unités fourragères [UF]) et en azote (matières azotées digestibles [MAD]) au cours du PEF. Pour calculer la couverture des besoins alimentaires des animaux, nous nous sommes appuyés sur les ouvrages de référence suivants : Rivière (1991), Le Thiec (1996) et Meyer et Denis (1999) pour estimer les besoins des animaux et les ingérés en aliments et fourrages ; Guérin et al. (1991) pour estimer les ingérés au pâturage en fonction des saisons. Nous avons calculé la couverture des besoins des animaux en UF et MAD, définie par le ratio (ingéré/besoins), qui est égal à $100 \%$ si le besoin est couvert, supérieur à $100 \%$ s'il y a excédent et inférieur à $100 \%$ en cas de déficit.

\section{Résultats}

\subsection{Tous les producteurs ont des projets d'élevage familiaux en tête}

Les résultats de l'enquête préliminaire ont montré que tous les producteurs avaient des perspectives d'évolution de leur élevage en tête et, bien souvent, un PEF en cours de préparation (Tab. 1). Chez les agriculteurs (exploitations principalement orientées vers l'agriculture et disposant de peu d'animaux), les perspectives concernaient surtout l'acquisition de BdT (PEF $\mathrm{BdT}$ ) et la constitution d'un petit noyau d'élevage, du fait de moyens financiers limités. Chez les agro-éleveurs (grandes exploitations en termes de surface et de cheptel), les perspectives concernaient la consolidation du troupeau de bovins : une partie pour la capitalisation sur pied et une autre pour la production d'animaux embouchés (PEF BàE, fréquents) ou pour la production de lait (PEF VL, rares). Chez les éleveurs (exploitations principalement orientées vers l'élevage disposant de peu de surfaces cultivées), les perspectives concernaient aussi l'accroissement du troupeau de bovins, avec une forte propension au développement de PEF 
Tableau 1. Caractéristiques des exploitations de l'enquête préliminaire, évolution du cheptel, type de PEF envisagés, évolution des infrastructures d'élevage et des pratiques de conduite souhaitées par les producteurs selon leur profil.

Table 1. Structural characteristics of farms, livestock evolution, projects pattern planned, evolution of the infrastructures of breeding and practices of control planned by producers according to their profile.

\begin{tabular}{|c|c|c|c|c|}
\hline Type d'exploitations & Année & Agriculteur $(n=15)$ & Agro-éleveur $(n=13)$ & Éleveur $(n=12)$ \\
\hline \multicolumn{5}{|l|}{ Caractéristiques des exploitations } \\
\hline Nombre de personnes à charge & 2013 & $12 \pm 9$ & $22 \pm 22$ & $16 \pm 6$ \\
\hline Surface cultivée (ha) & 2013 & $11,2 \pm 7,7$ & $18,5 \pm 15,1$ & $3,4 \pm 1,6$ \\
\hline Nombre de bovins $(n)$ & 2013 & $5 \pm 3$ & $33 \pm 23$ & $55 \pm 25$ \\
\hline Capacité d'autofinancement & 2013 & + & +++ & ++ \\
\hline \multirow{2}{*}{ Bovins d'élevage } & 2013 & $2 \pm 2$ & $28 \pm 22$ & $53 \pm 25$ \\
\hline & Futur & $8 \pm 9$ & $85 \pm 60$ & $100 \pm 71$ \\
\hline PEF BdT en préparation & 2013 & Très fréquents & Fréquents & Fréquents \\
\hline PEF BàE en préparation & 2013 & Très rares & Fréquents & Fréquents \\
\hline PEF VL en préparation & 2013 & Très rares & Rares & Très fréquents \\
\hline \multirow{3}{*}{ Fosses fumières $(\mathrm{Nb})$} & 2013 & $2 \pm 1$ & $2 \pm 1$ & $1 \pm 1$ \\
\hline & Futur & $3 \pm 1$ & $3 \pm 3$ & $1 \pm 1$ \\
\hline & 2013 & $0,2 \pm 0,2$ & $0,1 \pm 0,2$ & $0,3 \pm 0,3$ \\
\hline Cultures fourragères (ha) & Futur & $0,3 \pm 0,3$ & $0,3 \pm 0,4$ & $0,5 \pm 0,5$ \\
\hline \multirow{2}{*}{ Tourteau de coton (kg/an/bovin) } & 2013 & $50 \pm 45$ & $23 \pm 22$ & $2 \pm 2$ \\
\hline & Futur & $29 \pm 25$ & $15 \pm 13$ & $11 \pm 17$ \\
\hline \multirow{2}{*}{ Fourrages (kg/an/bovin) } & 2013 & $339 \pm 516$ & $42 \pm 45$ & $14 \pm 25$ \\
\hline & Futur & $241 \pm 266$ & $37 \pm 68$ & $26 \pm 45$ \\
\hline \multirow{2}{*}{ Soins vétérinaires (FCFA/an/bovin) } & 2013 & $4142 \pm 3602$ & $2002 \pm 935$ & $1626 \pm 889$ \\
\hline & Futur & $1496 \pm 1085$ & $1250 \pm 1226$ & $1253 \pm 1077$ \\
\hline
\end{tabular}

PEF : projet d'élevage familial ; BdT: bovins de trait; BàE : bovins à l'engraissement ; VL : vaches laitières ; Nb : nombre ; $1 €=655,957$ FCFA

laitiers (PEF VL), et parfois aussi d'embouche bovine (PEF BàE).

Pour soutenir ces PEF, les producteurs envisageaient des améliorations des infrastructures d'élevage (plateformes à fourrages et fosses fumières) et un développement des cultures fourragères $(+0,1$ à $+0,3$ ha par rapport à aujourd'hui). Néanmoins, l'enquête a mis en évidence une tendance à la baisse des apports d'intrants par tête de bétail dans le futur (en aliment, en fourrage, en soins), ce qui dénote, dès le stade de l'intention du PEF, une maîtrise approximative des stratégies nécessaires à l'intensification des productions animales (seuls les éleveurs prévoient d'augmenter les apports d'aliments et de fourrages par tête, mais ils restent à des niveaux très bas).

\subsection{La majorité des projets d'élevage familiaux échouent avant leur mise en place}

Dans les exploitations suivies, la moitié des PEF BdT et BàE n'ont pas été mis en œuvre ; la totalité des PEF VL ont été réalisés (Tab. 2).
Les résultats montrent que les $\mathrm{PEF}$ BdT non réalisés (Tab. 2) concernent des exploitations modestes, disposant de peu de moyens financiers et humains, qui avaient préparé des stocks de fourrage et d'aliments par tête de bovin plus faibles que dans les PEF BdT réalisés, et qui n'avaient pas produit de cultures fourragères, ce qui entraînait une moins bonne couverture des besoins des animaux en UF et MAD.

Les PEF BàE non réalisés (Tab. 2) concernent aussi des exploitations disposant de moyens financiers et humains plus modestes que celles ayant un PEF réalisé. Dans le cas des PEF BàE non réalisés, les stocks d'aliments prévisionnels étaient très faibles, ce qui était peu compatible avec la réussite d'une activité d'embouche. La couverture des besoins des animaux en UF et en MAD était aussi moins bonne que dans les PEF réalisés. Enfin, les porteurs de PEF non réalisés escomptaient des marges trop élevées, ne tenant pas compte de la réalité du marché, contrairement à ceux qui ont mené leur PEF au bout.

Dans le cas des PEF BdT et BàE, la non-mise en œuvre pouvait donc s'expliquer par un manque de préparation, un défaut de calibrage et un manque de réalisme économique. 
Tableau 2. Caractéristiques des exploitations suivies, des PEF prévisionnels et des PEF réalisés.

Table 2. Characteristics of monitored farms, estimated family livestock projects (FLP) and FLPs carried out.

\begin{tabular}{|c|c|c|c|c|c|c|}
\hline Réalisation des PEF & \multicolumn{6}{|c|}{ PEF } \\
\hline Nombre d'actifs & $7 \pm 2$ & $3 \pm 0$ & $15 \pm 4$ & $6 \pm 1$ & 11 & $12 \pm 9$ \\
\hline Capacité financière & ++ & + & +++ & ++ & + & +++ \\
\hline \multicolumn{7}{|l|}{ Nombre d'animaux } \\
\hline $\mathrm{P}$ & $3 \pm 2$ & $4 \pm 2$ & $7 \pm 1$ & $6 \pm 3$ & 5 & $5 \pm 0$ \\
\hline $\mathrm{R}$ & $3 \pm 2$ & $\mathrm{X}$ & $4 \pm 1$ & & 3 & $10 \pm 3$ \\
\hline \multicolumn{7}{|c|}{ Stock de fourrages/UBT (kg) } \\
\hline $\mathrm{R}$ & $212 \pm 82$ & $\mathrm{X}$ & $223 \pm 10$ & $\mathrm{X}$ & 243 & $365 \pm 413$ \\
\hline \multicolumn{7}{|c|}{ Surface de culture fourragère (ha) } \\
\hline $\mathrm{P}$ & $0,8 \pm 0,3$ & $0,8 \pm 0,4$ & $1 \pm 0$ & $1 \pm 0$ & 0,50 & $1 \pm 0$ \\
\hline $\mathrm{R}$ & $0,7 \pm 0,1$ & $\mathrm{X}$ & $0,8 \pm 0,4$ & $\mathrm{X}$ & 0,50 & $1 \pm 0$ \\
\hline \multicolumn{7}{|c|}{ Couverture besoin UF/UBT (\%) } \\
\hline $\mathrm{P}$ & $124 \pm 29$ & $114 \pm 12$ & $151 \pm 56$ & $107 \pm 6$ & 87 & $76 \pm 7$ \\
\hline $\mathrm{R}$ & $108 \pm 9$ & $\mathrm{X}$ & $82 \pm 8$ & $\mathrm{X}$ & 111 & $110 \pm 19$ \\
\hline \multicolumn{7}{|c|}{ Couverture besoin MAD/UBT (\%) } \\
\hline $\mathrm{P}$ & $211 \pm 25$ & $209 \pm 23$ & $279 \pm 28$ & $220 \pm 36$ & 70 & $57 \pm 9$ \\
\hline $\mathrm{R}$ & $143 \pm 7$ & $\mathrm{X}$ & $182 \pm 81$ & $\mathrm{X}$ & 95 & $98 \pm 40$ \\
\hline \multicolumn{7}{|l|}{ Marge/UBT (kFCFA) } \\
\hline $\mathrm{P}$ & - & - & $74 \pm 76$ & $145 \pm 26$ & 5 & $42 \pm 12$ \\
\hline
\end{tabular}

PEF : projet d'élevage familial ; BdT : bovins de trait ; BàE : bovins à l'engraissement ; VL : vaches laitières ; Nb : nombre ; UBT : unité de bétail tropical $=$ bovin de $250 \mathrm{~kg} ;+$ : faible $;++$ : moyenne $;+++$ : forte $; \mathrm{P}:$ prévisionnel $; \mathrm{R}:$ réalisé $; n:$ nombre ; Bv : bovin ; UF : unité fourragère ; MAD : matières azotées digestibles ; - : non concerné ; X : PEF non réalisé ; $1 €=655,957$ FCFA.

Dans le cas des PEF VL (Tab. 2), l'association du PEF à une activité de transformation du lait à la ferme augmente, dans une très forte proportion, la valorisation économique du projet (marge élevée). Dans les PEF VL, les objectifs prévisionnels de cultures fourragères ont été tenus.

\subsection{Maîtrise approximative de l'alimentation dans les projets d'élevage familiaux réalisés}

Sur la durée totale des PEF, pour les PEF BdT et BàE, la couverture des besoins en énergie comme en matières azotées était largement excédentaire dans les PEF prévisionnels, puis a convergé vers $100 \%$ dans les PEF réalisés (Tab. 2), en raison des ajustements opérés par les producteurs pendant la mise en œuvre. Concernant les PEF VL, la couverture des besoins était déficitaire dans les PEF prévisionnels, puis a convergé vers l'équilibre (100 \%) dans les PEF réalisés, pour la même raison que précédemment.

Les suivis ont permis de mettre en évidence d'importants déséquilibres alimentaires (déficits ou excédents) pendant le déroulement des PEF, comme le montre la Figure 1 : besoins des animaux non couverts aux périodes critiques dans les PEF $\mathrm{VL}$ et BdT, ou déséquilibre alimentaire (excès de MAD) dans les rations des BàE. L'équilibre alimentaire apparent, que l'on a observé sur la durée totale des PEF, masquait en réalité des périodes de déficits et des périodes d'excédents, se compensant plus ou moins au final, mais qui ont néanmoins pénalisé la réussite des PEF.

La Figure 1 montre que dans les PEF BdT, les besoins des animaux étaient stables jusqu'en mars, puis on observe une augmentation des besoins à partir de mai, due aux travaux champêtres. Concernant la couverture des besoins en UF et en $\mathrm{MAD}$, on observe un excédent entre janvier et avril, qui se réduit peu à peu, avec une évolution vers une situation déficitaire vers la fin de la saison sèche (avril), en raison de l'épuisement de stocks de résidus de cultures. Pour compenser cette pénurie fourragère, les producteurs ont eu recours aux compléments alimentaires, principalement de la drêche de sorgho. Cet aliment étant pauvre en énergie, les compléments distribués ne permettaient pas de couvrir l'augmentation subite 


\section{PEF BdT}

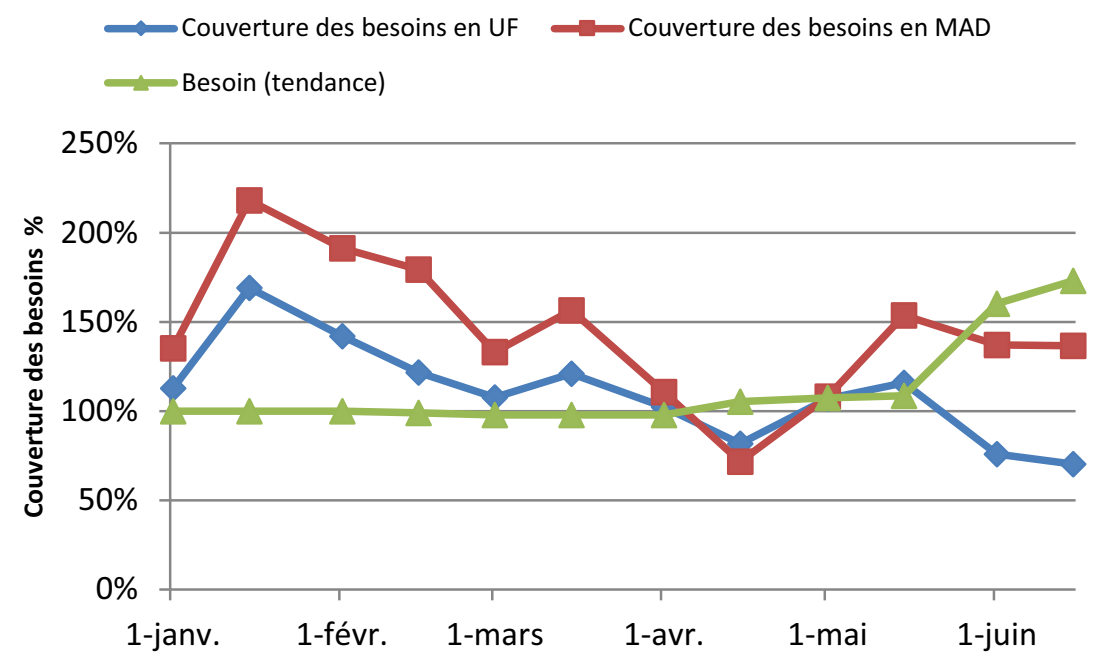

\section{PEF BàE}
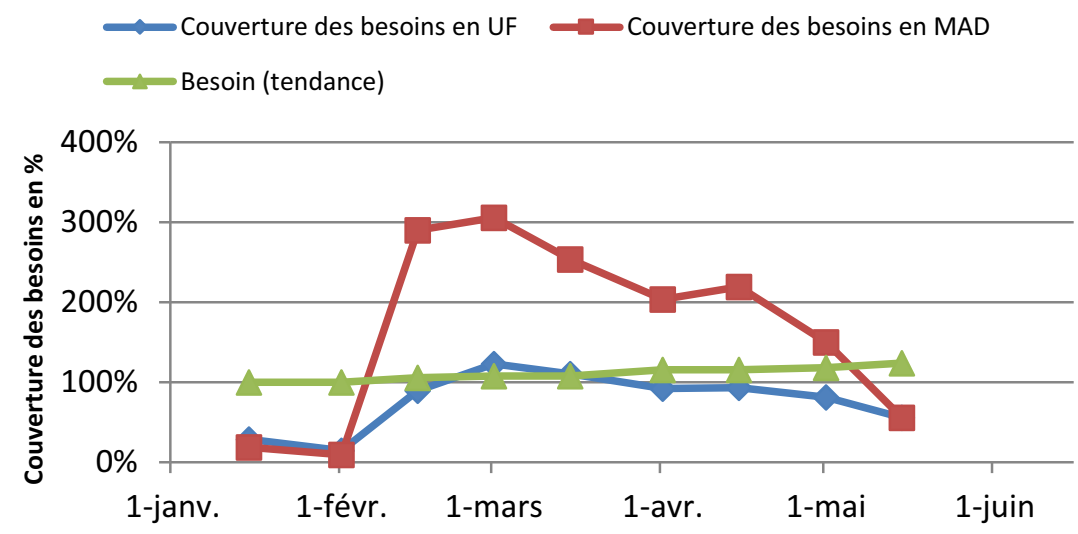

$1 \mathrm{~b}$

\section{PEF VL}

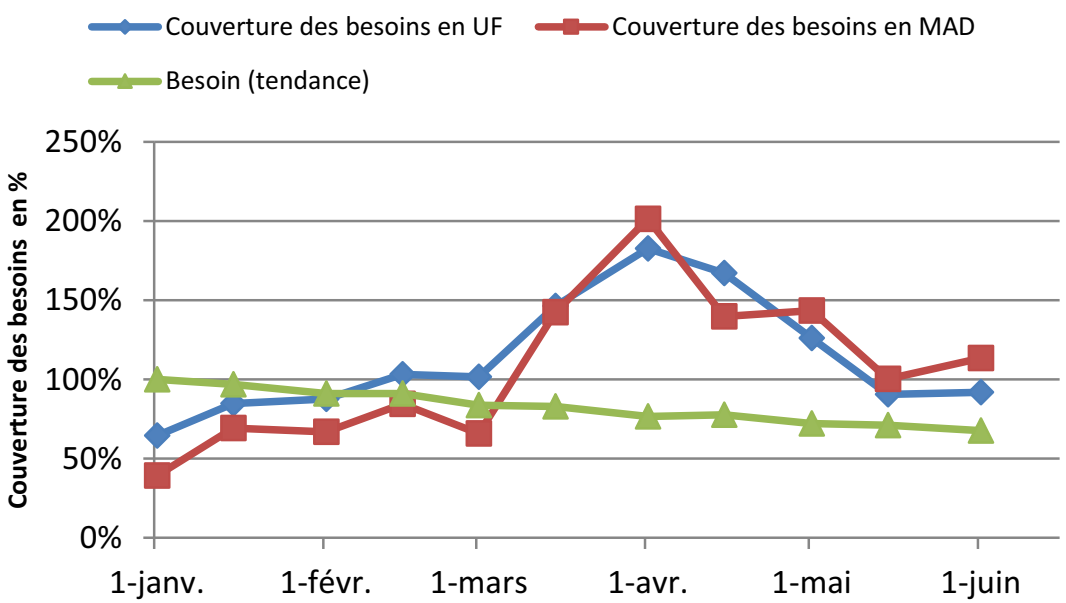

1a 
des besoins en énergie en début de campagne agricole (mai, juin).

Pour les PEF BàE, la Figure 1 montre que l'évolution des besoins était proportionnelle à l'augmentation de poids des bovins. Concernant la couverture des besoins alimentaires, on observe d'abord un déficit en début d'exécution du projet parce que la ration était basée sur des pailles. Puis à partir de mi-février, on constate un excédent très important en MAD parce qu'à cette période les éleveurs ont eu recours à des distributions massives de tourteau de coton (aliment très riche en azote). Enfin, en phase de finition des animaux (mai), la couverture des besoins n'est pas satisfaite, ce qui n'est pas idéal pour préparer la commercialisation des animaux.

Pour les PEF VL, la plupart des vaches ayant mis bas en octobre-novembre de l'année précédente, les pics de lactation étaient passés, ce qui explique une baisse régulière des besoins durant l'exécution du PEF (Fig. 1). En ce qui concerne la couverture des besoins alimentaires, on a constaté un déficit entre janvier et fin février, parce qu'à cette période, où les besoins étaient encore élevés (on était encore proche du pic de lactation), l'offre alimentaire était basée sur le pâturage et les résidus de cultures pauvres (pailles de maïs et sorgho), sans complémentation en légumineuses et en aliments concentrés. Entre mars et mai (saison sèche chaude), on a observé un léger excédent dû à un renforcement des rations en son de maïs et farine basse de riz.

\section{Discussion}

\subsection{Des problèmes de préparation, de calibration et de maîtrise de l'alimentation dans les PEF}

L'étude montre que les producteurs ont en tête des PEF qui diffèrent selon les objectifs et les besoins de leurs exploitations, et selon leur capacité de travail et d'autofinancement (car l'accès au crédit est limité). Ce constat n'est pas spécifique aux PEF bovins de la zone d'étudiée, comme l'ont montré les études sur les stratégies des exploitations familiales (McDermott et al., 2010 ; Udo et al., 2011) et sur le rôle-clé de la capacité d'autofinancement dans la réussite des projets des producteurs (Vall et al., 2007).

Durant la préparation du PEF, l'étude montre qu'un nombre important de producteurs rencontrent des difficultés en termes de production de fourrages, de constitution de stocks de fourrages suffisants, d'acquisition d'aliments, d'ajustement de la taille du troupeau aux ressources alimentaires nécessaires, et qu'ils escomptent des revenus qui ne correspondent pas à la réalité du marché. Ces difficultés révèlent un besoin de renforcement des capacités des producteurs :

- dans le domaine technique sur la production et la conservation des fourrages (Roothaert et al., 2003) ;

- dans la gestion de l'élevage familial (Awono Bessa et al., 2003 ; Faure et al., 2011) ;

- dans l'accès à l'information sur les marchés des produits animaux (IFAD, 2001).

Durant la mise en œuvre, on note de fréquents déséquilibres entre les besoins des animaux et l'offre alimentaire, ce qui montre que les producteurs ont des difficultés d'ajustement de l'offre alimentaire à l'évolution des besoins des animaux. On voit bien que la planification de l'alimentation sur la base des estimations des besoins des animaux affectés au projet est une tâche difficile, pour laquelle les producteurs ne semblent pas encore être préparés de manière adéquate. Or, les objectifs de production ne peuvent pas être atteints sans une bonne gestion de l'alimentation des animaux en fourrages et compléments alimentaires. Comme le précisent Coléno et Duru (1998), il est nécessaire de définir à l'avance la production fourragère et un calendrier d'utilisation du fourrage dans la mise en place du projet d'élevage. Ces difficultés d'ajustement pourraient être résolues par l'utilisation d'outils de gestion des rations des animaux, à condition qu'ils soient adaptés aux conditions locales, qu'ils ne demandent pas trop de données d'entrée et qu'ils proposent des sorties facilement compréhensibles pour les producteurs. Les outils existants, mis au point pour les milieux tempérés, comme INRAtion (Agabriel et al., 1999), ne répondent pas à ces critères, ce qui rend leur utilisation difficile pour ce type de PEF.

\subsection{Proposition d'une démarche de co-conception}

Ces difficultés ne concernent pas que les producteurs de l'Ouest du Burkina Faso, mais la grande majorité des exploitations de polyculture élevage des zones de savanes sèches et subhumides d'Afrique de l'Ouest et du Centre, c'està-dire des millions d'exploitations (Gafsi et al., 2007); la réussite de leurs PEF pourrait être améliorée si ces derniers étaient mieux accompagnés dans leur préparation et dans leur réalisation.

Ce constat et les difficultés observées chez les producteurs au cours des étapes de préparation et de mise en œuvre des PEF nous ont donc conduits à réfléchir à l'élaboration d'une méthode de co-conception des PEF. Elle viserait à aider le producteur à réussir un projet d'élevage en l'ajustant progressivement, en même temps qu'il apprendrait à le piloter, se convaincrait de son intérêt, et réorganiserait progressivement le travail et les moyens de production. Elle serait mise en œuvre dans une optique de conception innovante «pas à pas » (Meynard et Dourmad, 2014), et impliquant fortement le producteur et le conseiller (Vall et al., 2016). Cette méthode de co-conception progressive serait basée sur les étapes suivantes, toutes réalisées en dialogue avec le porteur du PEF :

- diagnostic initial : analyse du PEF initial (repérage des objectifs, des forces et des faiblesses);

- recherche d'options d'amélioration et formulation d'un PEF révisé : réduction des faiblesses initiales et préparation du PEF (planification, stratégie d'alimentation des animaux et stratégie de valorisation des produits);

- mise en œuvre et ajustements : suivi de la mise en œuvre du PEF et ajustement de l'alimentation à l'aide d'un outil simple (Encadré 1);

- évaluation et perspectives : évaluation des résultats afin de dégager des enseignements pour les futurs PEF. 
Encadré 1. Altrop, un outil permettant d'évaluer la couverture des besoins alimentaires des bovins.

Altrop, a tool for assessing the feeding needs of cattle.

Un calculateur, dénommé Altrop (non publié) a été élaboré sur Excel. Il permet d'ajuster l'offre alimentaire (composée de pâturages, de fourrages et d'aliments), proposée à un lot de bovins affectés à une production particulière (énergie agricole, production de viande ou de lait) aux besoins de ces derniers en énergie (unités fourragères [UF]), en matières azotées digestibles (MAD) et en sels minéraux ( $\mathrm{Ca}$ et $\mathrm{P})$. Altrop a permis de gérer l'alimentation de lots de bovins en prenant en compte la logique des pratiques d'alimentation des éleveurs, tout en intégrant le système et les références zootechniques du Manuel d'alimentation des ruminants domestiques en milieu tropical (Rivière, 1991).

En amenant les producteurs à s'interroger sur leurs PEF pour en déceler les faiblesses et réfléchir aux solutions possibles, cette méthode de co-conception devrait contribuer à renforcer la viabilité et la durabilité des PEF. En outre, cette méthode étant fondée sur un dialogue permanent entre le conseiller et le producteur, permettrait d'élaborer des références originales sur les stratégies et les modes de conduite des systèmes d'élevage familiaux.

\section{Conclusion}

Cette étude des PEF bovins dans les exploitations de polyculture élevage de l'Ouest du Burkina Faso a permis de mettre en évidence leurs faiblesses et les raisons qui font que très souvent les producteurs ne peuvent pas les mettre en place. L'une des principales causes de l'échec de mise en œuvre des PEF projetés est leur manque de préparation et leur défaut de calibrage. Pour ceux qui passent à l'étape de réalisation, le manque de maîtrise de l'alimentation affaiblit leur rentabilité. Face à cette situation, il est nécessaire d'élaborer une démarche de co-conception pas à pas de $\mathrm{PEF}$ pour améliorer leur réussite, méthode qui pourrait avoir des applications sur l'ensemble des zones de savanes d'Afrique de l'Ouest et du Centre. Enfin, nos résultats montrent que le rendement économique des PEF est relativement faible. L'incorporation d'une étape de transformation des produits dans les PEF permettrait probablement d'améliorer cette rentabilité.

\section{Références}

Agabriel J, Champciaux P, Espinasse C. 1999. INRAtion-PrevAlim 2.7 : logiciel de rationnement des ruminants. Dijon, France: INRACNERTA.

Alary V, Duteurtre G, Faye B. 2011. Élevages et sociétés : les rôles multiples de l'élevage dans les pays tropicaux. INRA Prod. Anim. 24: 145-156.

Awono Bessa C, Havard M, Assana M. 2003. Le conseil à l'élevage : une démarche pour accompagner l'intégration de l'élevage à l'agriculture dans les exploitations agricoles du Nord-Cameroun? In : Jamin JY, Seiny Boukar L, Floret C, eds. Savanes africaines : des espaces en mutation, des acteurs face à de nouveaux défis. Actes du colloque, mai 2002, Garoua, Cameroun. N'Djamena, Tchad / Montpellier, France: Prasac / Cirad, https://www.hal.archivesouvertes.fr/hal-00143068/document.

Club du Sahel. 2008. Élevage et marché régional au Sahel et en Afrique de l'Ouest. Potentialités et défis. Paris: CSAO-OCDE, CDAO.

Coléno FC, Duru M. 1998. Gestion de production en systèmes d'élevage utilisateurs d'herbe : une approche par atelier. Etudes Rech. Syst. Agraires Dév. 31: 45-61.

De Ridder N, Sanogo OM, Rufino MC, van Keulen H, Giller KE. 2015. Milk: the new white gold? Milk production options for smallholder farmers in Southern Mali. Animal 9(7): 1221-1229, DOI: $10.1017 /$ S1751731115000178.

Douxchamps S, Van Wijk MT, Silvestri S, Moussa AS, Quiros C, Ndour NYB, et al. 2015. Linking agricultural adaptation strategies, food security and vulnerability: evidence from West Africa. Reg. Environ. Change 1-13, DOI: 10.1007/s10113-015-0838-6.

FAO, FIDA, PAM. 2013. L'état de l'insécurité alimentaire dans le monde 2013. Les multiples dimensions de la sécurité alimentaire. Rome: FAO.

Faure G, Desjeux Y, Gasselin P. 2011. Revue bibliographique sur les recherches menées dans le monde sur le conseil en agriculture. $\mathrm{Cah}$. Agric. 20: 327-342, DOI: 10.1684/agr.2011.0510.

Gafsi M, Dugué P, Jamin JY, Brossier J (coord.). 2007. Exploitations agricoles en Afrique de l'Ouest et du Centre. Versailles, France: Quae.

Guérin H, Friot D, Mbaye N, Richard D. 1991. Alimentations des ruminants domestiques sur pâturages naturels : étude méthodologique dans la région du Ferlo au Sénégal. Montpellier: IEMVT.

IFAD. 2001. Rural Poverty Report 2001: the challenge of ending rural poverty. Oxford: Oxford University Press.

Kagone H. 2001. Profil fourrager Burkina Faso. Rome: FAO.

Kiéma A, Nianogo AJ, Ouédraogo T, Somda J. 2008. Valorisation des ressources alimentaires locales dans l'embouche ovine paysanne: performance technico-économiques et options de diffusion. Cah. Agric. 17: 23-27, DOI: 10.1684/agr.2008.0154.

Le Thiec G. 1996. Agriculture africaine et traction animale. Montpellier: Cirad (Techniques).

Lebacq T, Baret PV, Stilman T. 2013. Sustainability indicators for livestock farming. A review. Agron. Sustain. Dev. 33: 311-327, DOI: $10.1007 / \mathrm{s} 13593-012-0121-\mathrm{x} 4$.

McDermott J, Staal SJ, Freeman HA, Herrero M, Van de Steeg JA. 2010. Sustaining intensification of smallholder livestock systems in the tropics. Livest. Sci. 130: 95-109, DOI: 10.1016/j.livsci. 2010.02.014.

Meyer C, Denis JP. 1999. Élevage de la vache laitière en zone tropicale. Montpellier: Cirad (Techniques).

Meynard JM, Dourmad JY. 2014. L'innovation en élevage : de nouvelles démarches pour de nouveaux enjeux. INRA Prod. Anim. 27-2: 77-88.

Ministère des Ressources animales (MRA). 2012. Rapport annuel d'activités (année 2011). Ouagadougou: ministère des Ressources animales.

Rivière R. 1991. Manuel d'alimentation des ruminants domestiques en milieu tropical. Paris: La Documentation française.

Roothaert R, Horne P, Stür W. 2003. Integrating forage technologies on smallholder farms in the upland tropics. Trop. Grassl. 37: 295-303.

Udo H, Aklilu H, Phong L, Bosma R, Budisatria I, Patil B, et al. 2011. Impact of intensification of different types of livestock production in smallholder crop-livestock systems. Livest. Sci. 139: 22-29, DOI: 10.1016/j.livsci.2011.03.020.

Vall E, Dugué P, Blanchard M. 2006. Le tissage des relations agriculture-élevage au fil du coton. Cah. Agric. 15(1): 72-79. 
Vall E, Djamen P, Havard M, Roesch M. 2007. Investir dans la traction animale : le conseil à l'équipement. Cah. Agric. 16(2): 93-100, DOI: 10.1684/agr.2007.0080.
Vall E, Chia E, Blanchard M, Koutou M, Coulibaly K, Andrieu N. 2016. La co-conception en partenariat de systèmes agricoles innovants. Cah. Agric. 25(1): 15001, DOI: 10.1051/cagri/2016001.

Cite this article as: Delma BJ, Bougouma-Yameogo V, Nacro HB, Vall E. 2016. Fragilité des projets d'élevage familiaux dans les exploitations de polyculture-élevage au Burkina Faso, Cah. Agric. 25: 35005. 